Christine Morgenstern*

\title{
Need for Speed: Die neue Beschleunigungs- freude im Strafverfahren am Beispiel der Untersuchungshaft
}

\author{
https://doi.org/10.1515/zfrs-2020-0004
}

Zusammenfassung: Sowohl nach nationalem wie nach europäischem Recht gelten für die Untersuchungshaft als ultima ratio der Verfahrenssicherung strenge Anordnungsvoraussetzungen, die von den Gerichten in einer komplexen Prüfstruktur festzustellen sind. Ebenso finden sich normative Verpflichtungen, Strafverfahren in Haftsachen zügig und gegenüber anderen Verfahren beschleunigt einer Klärung zuzuführen. Der Beitrag erhellt, dass in der Praxis zunehmend das eine Prinzip gegen das andere ausgespielt wird und das Bedürfnis, Verfahren schnellstmöglich zu erledigen, die Notwendigkeit einer gründlichen Prüfung der Haftvoraussetzungen überlagert. Augenfällig ist dies für Verfahren eher leichter Kriminalität gegen sozial marginalisierte ausländische Tatverdächtige. Dieser länderübergreifende Befund wird anhand von Ergebnissen einer vergleichenden empirischen Studie zur Untersuchungshaftvermeidung in sieben europäischen Staaten dargestellt. Die beobachtete neue Beschleunigungsfreude lässt sich Erscheinungen der Ökonomisierung und Marginalisierung des Rechts zuordnen, die durch gesetzlich verankerte oder kriminalpolitisch geforderte Möglichkeiten, Strafverfahren zu „effektivieren“, begünstigt werden.

Abstract: In order to comply with the requirements by domestic and European law, pre-trial detention als ultimum remedium for securing the criminal proceedings can only be ordered by the courts following strict rules. At the same time, the law requires them to conduct these proceedings in a speedy manner. This contribution sheds light on current practices in which the two sometimes conflicting procedural principles are played off against each other and the aim to handle criminal proceedings speedily overrides the need to thoroughly examine the prerequisites for pre-trial detention. Proceedings against socially marginalised foreign offenders for petty offences are particularly affected. Findings from an empirical study on pre-trial detention in seven European states

\footnotetext{
*Korrespondezautorin: Prof. Dr. Christine Morgenstern, Professur Strafrecht und Geschlechterforschung, Freie Universität Berlin, Fachbereich Rechtswissenschaft, Van’t-Hoff-Str. 8, 14195 Berlin, Deutschland, E-Mail: christine.morgenstern@fu-berlin.de
}

Ә Open Access. (c) 2021 Morgenstern, publiziert von De Gruyter. (c) BY Dieses Werk ist lizensiert unter einer Creative Commons Namensnennung 4.0 International Lizenz. 
illustrate that this is a problem across borders. The observed new culture of speed can be attributed to wider concepts of the economisation and marginalisation of the law.

Keywords: speedy procedure, pre-trial detention, marginalised offenders

\section{„Hauptsache, es geht schnell“ - Beschleunigungsgrundsatz, Untersuchungshaft und die Marginalisierten des Rechts}

Ein menschengerechtes Strafverfahren sorgt dafür, dass über die gegen eine Person ,erhobene strafrechtliche Anklage ... in einem fairen Verfahren, ... innerhalb angemessener Frist verhandelt wird“, so will es Art. 6 der Europäischen Menschenrechtskonvention (EMRK). Verkürzt wird oft vom „Beschleunigungsgebot“ gesprochen, obwohl es nicht um Schnelligkeit per se, sondern um die Beachtung einer angemessenen Dauer geht. Sowohl nach nationalem wie nach europäischem Recht gibt es außerdem die staatliche Verpflichtung, nur unter strengen Bedingungen einer Person die Freiheit zu entziehen. Das gilt namentlich für diejenigen, die wegen des Verdachts eine Straftat begangen zu haben, in Untersuchungshaft genommen werden sollen: Hier sind in einer komplexen Prüfstruktur die Voraussetzungen nicht nur für das Bestehen eines Tatverdachts, sondern auch für das Bestehen eines der enumerativ aufgezählten Haftgründe und der Verhältnismäßigkeit einer solchen Haft festzustellen. Aus diesen beiden Grundsätzen ergibt sich das besondere Beschleunigungsgebot für Verfahren, in denen Untersuchungshaft vollstreckt wird - wird das Verfahren nicht angemessen zügig geführt, dauert die Untersuchungshaft also länger als notwendig, wird gegen dieses Gebot verstoßen.

Eine vergleichend angelegte qualitative Studie beschäftigte sich mit der Nutzung der Untersuchungshaft in sieben europäischen Staaten - wird sie tatsächlich als letztes Mittel, als ultima ratio der Verfahrenssicherung genutzt? Für das Fragenprogramm der Studie spielten temporale Aspekte eine wesentliche Rolle. Zum einen werden überlange Haftdauern als Problem vielerorts diskutiert, zum anderen werden aber auch eine allgemeine Überlastung der Strafgerichte und erheblicher zeitlicher Druck, gerade auch bei Haftsachen, berichtet. Im Lauf des Projekts wurde deutlich, dass die Akteurinnen und Akteure in der Justiz den Kampf mit der Zeit durchaus aufnehmen. Aus diesen Beschleunigungsstrategien ergab sich aber die Frage, inwieweit hierbei Gründlichkeit und Verhältnismäßigkeit der Entscheidungen auf der Strecke bleiben. Augenfällig ist dies für Verfahren 
eher leichter Kriminalität gegen sozial marginalisierte ausländische Tatverdächtige, exemplarisch dargestellt in einem der in Deutschland geführten Interviews:

Interviewerin: „Mir sind jetzt in Haftverhandlungen häufiger Fälle begegnet, in denen sagen wir - ein obdachloser polnischer Alkoholiker zum soundsovielten Mal einen Energydrink, eine Packung Fleischsalat und eine Flasche Weinbrand im Supermarkt geklaut hat; der Wert immer unter 25 Euro. Fällt Ihnen dazu etwas ein?“

RichterIn, Interview 14: „Nein. Mit fällt dazu nur ein, dass das Verfahren möglichst schnell stattfinden muss. ... Da ist dann Untersuchungshaft nicht zu vermeiden, sondern nur $\mathrm{zu}$ kürzen. Was Anderes fällt mir da nicht ein.“

In der DETOUR-Studie ging es in erster Linie um Untersuchungshaftvermeidung und geeignete Alternativen. ${ }^{1}$ Länderübergreifend ergab sich jedoch ein Zusammenhang zwischen Beschleunigungstendenzen und der Untersuchungshaft - oft wurde argumentiert, schnell geführte Verfahren trügen zwar nicht zur Vermeidung, immerhin aber zur Verkürzung der Untersuchungshaft bei. Daraus ergab sich der Verdacht, dass in manchen Fällen, d.h. vor allem bei den genannten sozial marginalisierten Betroffenen, das Beschleunigungsprinzip gegen andere Beschuldigtenrechte, namentlich Unschuldsvermutung und Freiheitsrechte, ausgespielt wird. Diese Wechselwirkung soll daher hier in ihrem rechtlichen und kriminalsoziologischen Kontext dargestellt und Erscheinungen der Ökonomisierung und Marginalisierung des Rechts zugeordnet werden. Überlegungen zu kriminalpolitischen Lösungsansätzen und zu weiterem Forschungsbedarf schließen den Beitrag ab.

\section{Das Recht auf ein zügiges Strafverfahren}

\section{Strafverfahrensgrundsätze}

Von der notwendigen „Effektivierung“ des Strafverfahrens ist oft die Rede (z.B. Bundesministerium der Justiz und für Verbraucherschutz 2015, Strafrechtliche Abteilung des Deutscher Juristentags, 1994). Die Forderung impliziert, dass es diese Effektivität noch nicht oder nicht mehr hat (zu den kriminalpolitischen

1 Das Projekt „Detour - Towards Pre-trial Detention as Ultima Ratio“ hatte die Haft- und Haftvermeidungspraxis in sieben europäischen Staaten (Deutschland, Belgien, Irland, Litauen, die Niederlande, Österreich und Rumänien) zum Gegenstand. Es wurde von der Europäischen Kommission gefördert; der Abschlussbericht wurde 2018 vorgelegt. Alle Ergebnisse sind abrufbar unter www.detour.at/irks. 
Bestrebungen später), sie verdeckt jedoch gleichzeitig, dass Effektivität erst dann gegeben ist, wenn mit sinnvollem Aufwand sinnvolle Ergebnisse erzielt werden (Röhl 2009: 114). Dies wiederum macht zumindest eine kurze Auseinandersetzung mit der Frage nach den angestrebten Ergebnissen und Zielen des Strafverfahrens notwendig.

Üblicherweise wird als Hauptziel des Strafverfahrens „eine in materiell-rechtlicher Hinsicht richtige und damit gerechte Entscheidung“ verstanden, „Wahrheit und Gerechtigkeit werden dadurch zu Leitprinzipien des Verfahrensrechts“ (Beulke \& Sowoboda, 2018: 3). Die Zitate sind bewusst aus einem populären Lehrbuch gewählt - auszubildenden Juristinnen und Juristen werden diese zentralen Werte vermittelt. Verbunden wird dies mit dem Hinweis auf widerstreitende, ggf. auch miteinander unvereinbare Ziele, die dementsprechend gegeneinander abzuwägen seien. Ungeachtet ob Lehrbuch, Kommentar oder wissenschaftliche Abhandlung findet sich dann stets der Topos des „Machbaren“: Was auch immer die Ziele sein mögen, notwendig ist jedenfalls ein funktionierender Apparat der Rechtsdurchsetzung. Oft folgt die Mahnung, die auch das Bundesverfassungsgericht akzeptiert: Die „Funktionstüchtigkeit der Strafrechtspflege“ müsse gewahrt bleiben (zurückgehend auf eine Entscheidung aus dem Jahr 1972, BVerfGE 33, 367, zuletzt vor allem in der Entscheidung zu den Absprachen im Strafverfahren, BVerfGE 133, 168); in der Regel findet sich dann auch ein Hinweis auf die Begrenztheit der Ressourcen.

Verbunden mit den $\mathrm{zu}$ erreichenden Zielen, bei denen die herrschende Rechtsdogmatik wohl davon ausgeht, dass auf ,diejenigen Elemente des Strafverfahrens, die der Wahrheitsfindung dienen, am wenigsten verzichtet werden kann“ (Weigend 2001: 271), sind die sog. Prozessmaximen oder Strafverfahrensgrundsätze. Sie werden als „charakteristischer Zug deutscher Rechtskultur insgesamt“ (Roxin \& Schünemann 2017: 66) hochgehalten. Zahl und Zuschnitt dieser Maximen variieren. Stets genannt ist jedoch das Beschleunigungsgebot als Bestandteil der Grundsätze der Durchführung des Verfahrens. Abgeleitet wird es aus verschiedenen Quellen, teilweise einfachrechtlich (aus den Vorschriften zur Hauptverhandlung, z. B. § 229 StPO) und auf der Ebene des Grundgesetzes aus dem Rechtstaatsprinzip. Vor allem aber ist der bereits zitierte Art. 6 Abs. 1 EMRK maßgeblich: ,Jede Person hat ein Recht darauf, daß über Streitigkeiten in Bezug auf ihre zivilrechtlichen Ansprüche und Verpflichtungen oder über eine gegen sie erhobene strafrechtliche Anklage von einem unabhängigen und unparteiischen, auf Gesetz beruhenden Gericht in einem fairen Verfahren, öffentlich und innerhalb angemessener Frist verhandelt wird.“ 


\section{Haftsachen}

Wer inhaftiert ist, hat einen nochmals betonten Anspruch auf zügige Bearbeitung seines Verfahrens. Dies ergibt sich aus verschiedenen Regelungen der deutschen Strafprozessordnung und aus Art. 5 Abs. 3 EMRK: „Jede Person, die nach Absatz 1 Buchstabe c von Festnahme oder Freiheitsentziehung betroffen ist, muß unverzüglich einem Richter oder einer anderen gesetzlich zur Wahrnehmung richterlicher Aufgaben ermächtigten Person vorgeführt werden; sie hat Anspruch auf ein Urteil innerhalb angemessener Frist oder auf Entlassung während des Verfahrens." Gestärkt wird dieser Anspruch durch Art. 5 Abs. 4 EMRK als Habeas Corpus-Vorschrift der EMRK, wonach „ein Gericht innerhalb kurzer Frist über die Rechtmäßigkeit der Freiheitsentziehung“ entscheiden muss, wenn dies beantragt wird. Es sind diese beiden Vorschriften, über die die Rechtsprechung zur Untersuchungshaft des Europäischen Gerichtshofs für Menschenrechte (im Folgenden: EGMR) einen großen Teil ihrer Wirksamkeit entfaltet (Morgenstern 2018: $187 \mathrm{ff}$.). Dabei betrachtet der EGMR ähnlich wie Teile der deutschen Rechtsdogmatik (z. B. Hassemer 1984: 40, Paeffgen 1986: 211) die Untersuchungshaft als prinzipiell zumutbares Sonderopfer für die verdächtige Person, die demnach die Haft als prozessuales Zwangsmittel bei Vorliegen der gesetzlichen Voraussetzungen hinzunehmen hat - aber eben nur, solange sie nicht „ein vernünftiges Maß überschritten hat, d. h. [ihr] eine größeres Opfer abverlangt hat, als nach den Umständen des Falles vernünftigerweise von einer Person, für die die Unschuldsvermutung gilt, erwartet werden darf.“2 Anerkannt ist ebenfalls, dass das Freiheitsrecht an Gewicht gewinnt und die Zumutbarkeit schwindet, je länger das Verfahren bzw. die Untersuchungshaft dauert, denn Grundrechtsschutz ist auch durch die Verfahrensgestaltung zu bewirken. ${ }^{3}$ Daraus schließt nicht nur der EGMR, sondern auch das Bundesverfassungsgericht, dass die Anforderungen an die Justizorganisation mit der Dauer der Untersuchungshaft steigen. ${ }^{4}$ Wenngleich beide Gerichte sich schwer tun, absolute Höchstgrenzen für die Länge der Untersuchungshaft zu nennen, ist die Begrenzung der Dauer der Untersuchungshaft doch ein ständig wiederkehrendes Thema und die Mahnung zu einem zügigen Verfahren in Haftsachen

2 EGMR, Urt. v. 27.6.1998 - 2122/64 (Wemhoff ./. Deutschland), para. 5 (As to the Law), meine Übers.

3 BVerfGE 20, 45 (49f.); maßgeblich in jüngerer Zeit BVerfG NJW 2006, S. 672.

4 So schon BVerfGE 15, 474 (480); aktueller BVerfG, Beschl. v. 30.7.2014 - 2 BvR 1457/14 m. w. N. zur Rechtsprechung (Leitsatz in NStZ-RR 2014, S. 314). 
findet sich beständig, auch in aus der Rechtsprechung des EGMR abgeleiteten Empfehlungen des Europarats. ${ }^{5}$

Anzumerken ist für die Zwecke dieses Beitrags, dass das Beschleunigungsgebot in Haftsachen immer und für alle Haftsachen gilt - unterhalb der in $\S 121$ StPO normierte zeitliche Grenze, nach der im Regelfall die Untersuchungshaft auf sechs Monate begrenzt ist und jedenfalls nur unter besondere Voraussetzungen verlängert werden kann, findet in Deutschland jedoch kaum eine förmliche Kontrolle über die Angemessenheit der Untersuchungshaftdauer statt (näher Morgenstern 2018: 538 ff.; Schlothauer, Weider \& Nobis 2016: $425 \mathrm{ff}$.).

\section{Kriminalpolitik und Reformdiskussionen}

Trotz dieser Standards, die der EGMR sowie in Deutschland das Bundesverfassungsgericht und die Oberlandesgerichte für zumutbare Haftdauern und deren Kriterien im Einzelfall aufgestellt haben, wird die Überlastung insbesondere der Landgerichte immer wieder als Grund für rechtsstaatswidrige Verzögerungen genannt. Da es aber verfassungswidrig ist, „[...] eine längere als die verfahrensangemessene Aufrechterhaltung des Haftbefehls nur deshalb in Kauf zu nehmen, weil der Staat es versäumt, seiner Pflicht zur verfassungsgemäßen Ausstattung der Gerichte zu genügen, “ (BVerfG, Beschl. v. 13.10.2016 - 2 BvR 1275/16, Rn. 45), muss in solchen Fällen der Haftbefehl aufgehoben werden. Wenn dies tatsächlich geschieht, bezweckt es auch eine Disziplinierung der Justiz und vor allem des Fiskus' und der Politik, die durch medial aufbereitete und in der Regel skandalisierte Haftentlassungen wegen Verfahrensverzögerungen aufgeschreckt werden (Morgenstern 2018: $544 \mathrm{ff}.)^{6}$

Insofern ist nicht nur das rechtsstaatliche Gebot, für zügige Verfahren zu sorgen, sondern auch die Sorge, von den Obergerichten eine verschleppte Bearbeitung nachgewiesen zu bekommen und ggf. beschuldigte Personen aus der Untersuchungshaft entlassen zu müssen, ein starker Motor für die Beschleunigung des Strafverfahrens in Haftsachen. Dabei ist aber der Verdacht nicht von der Hand

5 Recommendation $\operatorname{Rec}(2006) 13$ on remand in custody, the conditions in which it takes place and the provision of safeguards against abuse.

6 Die Justiz ist deutlich bemüht, die Verantwortung der staatlichen Sparpolitik zuzuweisen, vgl. z. B. einen der Leitsätze aus OLG Bremen, Strafverteidiger 2016: 824: „Die mit der Haftprüfung betrauten Gerichte haben den Grundrechtsschutz der Betroffenen zu verwirklichen. Danach sind Haftentlassungen unvermeidbar, wenn es zu Verfahrensverzögerungen kommt, weil der Staat seiner Verpflichtung nicht nachkommt, die Justiz mit den erforderlichen personellen und sächlichen Mitteln auszustatten.“ 
$\mathrm{zu}$ weisen, dass der ins Feld geführte Beschleunigungsgrundsatz inzwischen ein Sammelbegriff für alle auf Beschleunigung gerichteten Interessen ist und von einer dem Schutz der Beschuldigten dienenden Prozessmaxime zum multifunktionalen rechtspolitischen Instrument verkommen ist. In der Diskussion wird das Dilemma zumeist eher beschrieben als konstruktiv bearbeitet- so beim Deutschen Juristentag 1994, dessen strafrechtliches Thema die Beschleunigung war: „Der Beschleunigungsgrundsatz hat sowohl dem staatlichen Interesse an einem möglichst zügigen, zeit- und kostensparenden Verfahren als auch dem Schutz des Beschuldigten vor vermeidbaren staatlichen Verfahrensverzögerungen sowie dem Interesse des Verletzten an einer schnellen Ahndung der ihn belastenden Straftat zu dienen.“ (Verhandlungen des 60. Deutschen Juristentags 1994, Beschluss II). Damals fand auch der Beschluss eine Mehrheit, dass - sofern keine Nachteile für die Rechtsfindung entstünden und keine rechtsstaatlichen Grundsätze preisgegeben würden - aus dem Beschleunigungsgrundsatz auch Einschränkungen von Beschuldigtenrechten abzuleiten seien, um den teils gegenläufigen Interessen in möglichst ausgewogener Weise Rechnung zu tragen.

Die oft sehr heftig geführte Diskussion dreht sich daher seit vielen Jahren um Themen wie die Straffung der Hauptverhandlung bzw. die Einschränkung von Beweisantragsrechten, der Richterablehnung, des Unmittelbarkeitsgrundsatzes und der Rechtsmittel. In einem Versuch, die „Eilkrankheit“ zu bekämpfen (Wohlers 2010: 2470) und die früheren Beschlüsse gleichsam einzufangen, widmete sich der Juristentag 2010 wieder dem Thema. Diesmal wurde beschlossen, dass die Beschleunigung des Verfahrens ,kein Wert an sich [sei], der es rechtfertigen würde, andere Verfahrensgarantien im Kern einzuschränken.“ (Verhandlungen des 60. Deutschen Juristentags 2010, Beschluss I. 2). Mehrheiten fanden sich auch für Beschlüsse, die auf die Notwendigkeit einer ausreichenden Ausstattung der Justiz und auf die Entkriminalisierung als Mittel zur Entlastung der Justiz hinwiesen - darauf wird zurückzukommen sein.

Die in jüngerer Zeit in Angriff genommene Reform zu einer „effektiveren und praxistauglicheren Ausgestaltung“ des Strafverfahrens, die eine Expertenkommission beschäftigte (Bundesministerium der Justiz und für Verbraucherschutz 2015) und $2017 \mathrm{zu}$ einem gleichnamigen Gesetz führte, stieß auf erhebliche Kritik (z. B: Singelnstein \& Derin 2017; von Seiten der Berufsverbände erwartbar am schärfsten durch die Anwaltschaft). ${ }^{7}$ Sie setzte diesbezüglich auf die alten Rezepte, d.h. neben neuen Befugnissen der Strafverfolgungsbehörden

7 Gesetzesbegründung und Stellungnahmen sind auf der Website des Bundesjustizministeriums zu finden: https://www.bmjv.de/SharedDocs/Gesetzgebungsverfahren/DE/Gesetz_zur_ effektiveren_und_praxistauglicheren_Ausgestaltung_des_Strafverfahrens.html, abgerufen am 1.10.2019. 
im Ermittlungsverfahren auch Regelungen, die (mutmaßliche) Verzögerungstaktiken der Verteidigung in der Hauptverhandlung, namentlich durch Ablehnungsund Beweisanträge, verhindern sollen. Ähnliches verspricht (oder droht an) der Koalitionsvertrag vom März 2018. ${ }^{8}$ Bemerkenswert ist im Zusammenhang mit dem Thema dieses Beitrags, dass in der Gesetzesbegründung von 2017 mehrfach auf den - lästigen, so meint man herauszulesen - Druck hingewiesen wird, dem die Gerichte durch den Beschleunigungsgrundsatz in Haftsachen unterliegen, ihre „äußerst anspruchsvolle Aufgabe wird für die Strafgerichte in der täglichen Praxis noch dadurch erschwert, dass sie sich einer dauerhaft hohen Arbeitsbelastung ausgesetzt sehen und insbesondere in Haftsachen an das Beschleunigungsgebot gebunden sind“ (Regierungsentwurf mit Begründung: 1, 11).

Verfolgt man die Diskussion wird aber deutlich, dass in der Regel an Umfangsverfahren, oft an schwierige Wirtschaftsstrafverfahren, gedacht wird, d.h. vor allem an Verfahren vor dem Landgericht. Das gilt für die rechtspolitische Diskussion, an der die Berufsverbände der Strafverteidigung maßgeblich beteiligt sind, die naturgemäß die oft unverteidigten Beschuldigten und Angeklagten in Verfahren vor den Amtsgerichten weniger im Blick haben. Das gilt aber auch und gerade für die wissenschaftliche Auseinandersetzung, die sich häufig mit den überlangen und komplexen Verfahren beschäftigt (z. B. Krehl \& Eidam 2006, Baumanns 2011, Pest 2017, Wittig 2019: 285 ff.; mit Blick auf Haftsachen Schlothauer, Weider \& Nobis 2016: 416 ff.; Ausnahmen bei Scheffler 2002, Lubitz 2019).

\section{Die dunkle Seite der Praxis: Beschleunigte Verfahren}

Dies bedeutet umgekehrt, dass die Masse der Verfahren in Bezug auf die Beschleunigung wenig Aufmerksamkeit erfährt: Ausweislich der Statistik Strafgerichte (Statistisches Bundesamt 2018) standen im Jahr 2017 rund 13.000 vor den Landgerichten erledigte Verfahren etwa 654.000 erledigte Verfahren vor den Amtsgerichten gegenüber. Obwohl also erstere statistisch betrachtet die Ausnahme sind, prägen sie die Diskussion um den Zustand des deutschen Strafverfahrens mit Blick auf temporale Aspekte. Gegenstand dieser Betrachtung ist jedoch auch und vor allem das andere Ende des Kontinuums, diejenigen Fälle also, die zwar massenhaft auftreten, jedoch vermeintlich simpel sind und deshalb schnell und rationell abgewickelt werden sollen. Sie werden an den Amtsgerichten, oft vor der Einzelrichterin verhandelt. Untersuchungshaft jedoch kann auch in diesen Fällen

8 https://www.bundesregierung.de/breg-de/themen/koalitionsvertrag-zwischen-cdu-csu-undspd-195906, abgerufen am 1.10.2019. 
angeordnet werden und wird es tatsächlich auch, denn die wenigen ausdrücklichen Verhältnismäßigkeitsbestimmungen der StPO (§ 113 StPO) greifen nicht.

Im Bereich der alltäglichen Bagatellkriminalität hat der Gesetzgeber seit den 1970er Jahren bekanntlich durchaus auch versucht, die Strafjustiz zu entlasten: Neben den Möglichkeiten der Verfahrenseinstellung nach den $§ \S 153 \mathrm{ff}$. StPO, die inzwischen den überwiegenden Teil der anklagefähigen Strafsachen erledigt (Heinz 2017: $91 \mathrm{ff}$. ), sind dies im Bereich des allgemeinen Strafrechts das schriftliche Urteilsverfahren nach den $\S \S 407 \mathrm{ff}$. StPO (Strafbefehl) und das beschleunigte Verfahren gem. $\$ \S 417 \mathrm{ff}$. StPO. Dieses Verfahren ist für einfach gelagerte Verfahren ohne Beweisschwierigkeiten gegen Erwachsene oder Heranwachsende vor den Amtsgerichten gedacht. Eine notwendige Verteidigung gibt es, wenn die konkrete Straferwartung mindestens sechs Monate beträgt; außerdem kann unter erleichterten Voraussetzungen eine besondere Form der Untersuchungshaft, die sogenannte Hauptverhandlungshaft angeordnet werden.

Im Schatten der vorgehend vorgestellten Diskussion besteht daher der Verdacht, dass sich die Justiz (bzw. ihr der Gesetzgeber) eine Entlastung an anderer Stelle verschafft - hier ist im Zusammenhang mit dem Beschleunigungsgrundsatz „die dunkle Seite der Praxis“ (Kühne 2010: 827), d.h. die Beschneidung der Beschuldigtenrechte durch beschleunigte Verfahrensformate vermutet und in Untersuchungen auch bestätigt worden (Ernst 2001, Lubitz 2010, vgl. auch Scheffler 2002). Allerdings sind jedenfalls ausweislich der statistischen Datenlage beschleunigte Verfahren in der Breite noch immer nicht sehr gebräuchlich: Die Statistik Strafgerichte gibt an, dass 2016 von den bereits genannten 654.537 erledigten Verfahren vor den Amtsgerichten nur $14.362 \mathrm{im}$ beschleunigten Verfahren (2,2\%) erledigt wurden. Dennoch scheint es so zu sein, dass diese Praxis sich an bestimmten Schwerpunkten - namentlich den Stadtstaaten und Ballungszentren im Ruhr- und Rhein-Main-Gebiet zunehmender Beliebtheit erfreut (oder dass statistisch nicht alle diese Verfahren erfasst werden). Jedenfalls findet sich in der Antwort auf eine Kleine Anfrage des Berliner Abgeordnetenhauses (Drucksache 18 / 11 110) des Berliner Senats die Angabe, dass im Jahr 2016 (neuere Zahlen liegen nicht vor) fast 2500 Verfahren nach $\S \S 417 \mathrm{ff}$. StPO erledigt wurden, davon immerhin 849 im sog. „besonders beschleunigten Verfahren“. Diese Verfahrensvariante bedeutet normalerweise eine Erledigung binnen 24 Stunden, sodass entweder am Tag der Festnahme oder am nächsten Tag verhandelt wird und trotz der Festnahme keine förmliche Vorführung vor eine Haftrichterin erfolgen muss. Ähnliche Expressverfahren gibt es in anderen Städten und Regionen.

Zur Illustration, was dies in der Praxis bedeuten kann, mag ein Bespiel aus der explorativen Anfangsphase des später noch beschriebenen DETOUR-Projekts dienen: Ich habe einige Tage am Amtsgericht Moabit in Berlin, Außenstelle Tempelhofer Damm, verbracht und mir dabei eine Reihe der „besonders beschleunig- 
ten Verfahren“ angesehen. Zumeist sind es Laden- und Straßendiebstähle, die verhandelt werden, durchaus auch jenseits der Geringwertigkeit (je nach Handtascheninhalt). Die Vorgeführten sind in der Regel keine Deutschen, in der Regel ist also eine Dolmetscherin notwendig und auch immer vorhanden. Bei einigen der Vorgeführten hätte es nahegelegen, ihre Verhandlungs- und Schuldfähigkeit zu überprüfen, da sie nach meiner Einschätzung drogenabhängig waren und/ oder unter Drogeneinfluss standen. Repräsentiert war, in den Worten der Amtsanwältin, die die Anklagevertretung an diesen Tagen übernahm, das „soziale Elend“ - in meinen Feldnotizen habe ich mir ihre Aussage ,wir verwalten hier das soziale Elend der Stadt“ notiert. Die Strafen sind milde, niedrige Geldstrafen sind häufig. Freiheitsstrafen, die zur Bewährung ausgesetzt werden, werden verhängt, wenn dieselben Personen recht rasch wiederkommen und die Wiederholungsgeschwindigkeit aus den Akten ersichtlich ist, oder sie sogar das Pech haben, wieder auf dieselbe Besetzung des Gerichts zu treffen. Die genannte Amtsanwältin und eine der befragten Richterinnen bekannten, dass letzteres sehr häufig faktisch dann doch zu Gefängnisaufenthalten führt, weil Geldstrafen nicht bezahlt werden (können) bzw. weil ein Bewährungswiderruf folgt. Was regelmäßig fehlt, ist Zeit für die Betroffenen, über ihre Verteidigung nachzudenken oder sie vorzubereiten ${ }^{9}$ und eine Würdigung der Täterpersönlichkeit mit den entsprechenden sozialen Hintergründen (entsprechend mit Blick auf Beispiele aus Bochum Ernst 2001 und Berlin und Brandenburg Lubitz 2010, vgl. zusammenfassend auch die Kritik von Scheffler 2002, der nachvollziehbar von einem „Sonderstrafrecht für Ausländer und Obdachlose“ spricht).

Es ist durchaus denkbar, dass diese schnellen Erledigungen helfen Untersuchungshaft zu vermeiden- in den beobachteten Fällen wäre bei schwierigerer Beweislage oder fehlendem Geständnis vermutlich ein Haftbefehl ergangen. Es gibt zu den beschleunigten Verfahren jedoch deutlich weniger Literatur als zu den überlangen Verfahren (Scheffler 2002, im Übrigen z. B. Schröer 1998, für Heranwachsende Putzke 2004) und wenig empirische Forschung (z. B. Aktenstudien zur Berliner und Brandenburger Praxis von Lubitz 2010 und zur Bochumer Praxis von Ernst 2001). Es mag daher sein, dass die Richterschaft noch immer eher zögerlich ist, die beschleunigte Form zu nutzen; ob und warum dies so ist bzw. was dies für die Untersuchungshaft bedeutet, ist jedoch unklar. Die Kriminalpolitik hätte dazu im Gegensatz jedenfalls gerne mehr schnelle Verfahren; insbesondere vom Modell „besonders beschleunigtes Verfahren“. Dies legen Medienberichte nahe, die mit anerkennendem Unterton von entsprechenden Modellprojekten und ihren

9 Sie werden auf das Recht, eine Verteidigerin oder einen Verteidiger hinzuzuziehen stets hingewiesen; mitunter geschieht dies auch, dann wird auch gewartet. 
Befürwortern berichten. ${ }^{10}$ Niedergelegt ist die Sympathie auch in einem Beschluss der Justizministerkonferenz von 2016, in dem rechtsstaatlich und straftheoretisch durchaus fragwürdig „[...] die Bedeutung einer Strafe, die ,auf dem Fuße folgt“ und die vor allem reisende Straftäter daran hindern kann, Verfahren zu verschleppen oder unterzutauchen, um sich der Strafverfolgung zu entziehen.“ bekräftigt wird. ${ }^{11}$

\section{Rechts- und kriminalsoziologische Deutungen der Beschleunigungsfreude im Strafverfahren}

\section{Die neue Kultur der Kriminalitätskontrolle und weitere kritische Ansätze}

Diese Befunde aus dem Recht bzw. der Rechtspraxis finden ihre Entsprechung in Konzepten und Narrativen der Rechts- und Kriminalsoziologie. Wenngleich über ihre Übertragbarkeit gestritten wird, sind hier vor allem anglo-amerikanische Befunde prägend, die sich unter dem Stichwort der „Kultur der Kontrolle“ (Garland 2001) unter anderem mit der Informalisierung und Ökonomisierung des Rechts beschäftigen; daneben spielt die Dichotomisierung zwischen „guten Bürgern“ und denjenigen, die Strafe verdienen, den „anderen“ / „Fremden“ / „Gefährlichen“ (Simon 2007) oder „Armen“ (Wacquant 2009) eine wichtige Rolle. Beiträge zur Kriminologie des Strafverfahrens bauen ebenfalls vielfach auf amerikanischer Forschung auf, hier ist besonders die Procedural-JusticeForschung zu nennen. Neuere Tendenzen in der europäischen kriminologischen Forschung interessieren sich für die Schattenseiten der Justizsysteme mit ihren formal garantierten Beschuldigten- und Betroffenenrechten: Es geht um

10 Z. B. „Turbo-Justiz“, Westdeutsche Zeitung vom 6.8.2015 (https://www.wz.de/nrw/ duesseldorf/kurzer-prozess-fuer-119-kriminelle_aid-29083323); „Kurzer Prozess für Kleinkriminelle“, Flensburger Tageblatt vom 16.12.2016 (https://www.shz.de/lokales/flensburger-tage blatt/kurzer-prozess-fuer-kleinkriminelle-flensburg-drueckt-aufs-tempo-id15564486.html); „Die Justiz drückt aufs Tempo“, Die Welt vom 7.2.2017 (https://www.welt.de/regionales/nrw/ article161873697/Mehr-beschleunigte-Strafverfahren.html); alle abgerufen am 1.10.2019. Diese Berichte haben durchweg einen anerkennenden Unterton. In anderen Presseberichten hingegen wird, wie bereits angemerkt, die Behäbigkeit der Justiz, gar, wenn sie zu Haftentlassungen führt, in der Regel scharf kritisiert.

11 Abrufbar auf der https://mdjev.brandenburg.de/media_fast/bb1.a.3663.de/top_ii.17_-_erfah rungen_mit_der_durchfuehrung_des_besonders_beschleunigten_verfahrens.pdf. 
eine Prozeduralisierung der Menschenrechte (Snacken 2015, Armstrong 2019, Morgenstern \& van Zyl Smit 2020) und die Frage nach dem wirklichen und wirksamen Zugang zum Recht in der „Access to Justice“-Forschung (Francioni 2007, Leitch 2013, European Union Agency for Fundamental Rights and Council of Europe 2016).

\section{Ökonomisierung der Justiz und Managerialism}

Für die Beschleunigungstendenzen sind vor allem die beobachtete Ökonomisierung des Rechts und als Teilaspekt die Ökonomisierung der Justiz relevant. Während die deutsche Rechtslehre und auch die Justizpraxis dem ökonomischen Denken im Recht skeptisch bis ablehnend gegenüberstehen, ist es in der utilitaristischen anglo-amerikanischen Rechtstradition nicht schwer unterzubringen (Röhl 2009, Wittig 2019). Die rechtstheoretische Fundierung, die die Frage nach der Effizienz des Justizsystems nicht nur zulässt, sondern laut stellt, hat ihre Entsprechung in den genannten kritischen Befunden der Rechtssoziologie: Danach ist die „neue Kultur der Kontrolle“ unter anderem durch Managementmaßnahmen und Leistungsindikatoren gekennzeichnet, die die Arbeit der Justizprofessionellen strengen Regeln unterwirft; die elektronische Datenverarbeitung und damit einhergehende Formen der Systemüberwachung und des controlling spielen eine früher nicht denkbare Rolle (Garland 2001: $18 \mathrm{f}$., 176 ff.). Begünstigt wird dies durch ein "ständiges Krisenempfinden“ (Garland 2001: 19), das eben nur durch ein systematisiertes Vorgehen und strenges Management beherrschbar erscheint (schon Bottoms 1995, Garland 1996) und damit der Ermessensausübung und der Einzelfallgerechtigkeit, die das justizielle Handeln an sich kennzeichnen, deutlich weniger Raum lässt.

Auch für Deutschland gibt es entsprechende Beobachtungen: Mit einer gewissen Freude am durch EDV und entsprechende Controllingprogramme erleichterten Messen und Vergleichen, der „Soziokalkulation“, folgt auch die deutsche Justizverwaltung mehr und mehr einer „kalkulativen Mentalität“ (Röhl 2009: 106). Hinzu kommen Sparsamkeitsbemühungen, die gerade von der öffentlichen Hand gefordert werden, sowie das Anerkenntnis der Tatsache, dass Recht eine Ressource ist, die knapp ist und verteilt werden muss. Dabei ist - wie eingangs erwähnt - viel von Effizienz die Rede, die sich jedoch nur an der Oberfläche, meist durch Erledigungszahlen, und nicht auf der Ebene der Qualität des Geleisteten messen lässt. Notorisch ist das Personalbedarfsberechnungssystem der Justiz (Pebb§sy), das in Zusammenarbeit mit einer Unternehmensberatung entstand und seit 2005 angewandt wird. Es geht von Durchschnittsbearbeitungszeiten in Strafsachen aus, die erstaunlich niedrig liegen; dennoch ist errechnet 
worden, dass die meisten Richterinnen und Richter sehr hohe Wochenarbeitszeiten haben (Schulte-Kellinghaus 2006). Wenngleich diese Berechnungsgrundlagen und mehr noch die Haushaltspolitik gerade in der Richterschaft auf erhebliche Kritik stieß, reagieren doch viele in der Justizpraxis mit „Arbeitsverdichtung“ (Schulte-Kellinghaus 2006: 170 f.) bzw. wählen eine der von Röhl (2009: 108f.) beschriebenen Strategien, die neben der Leistungsverzögerung auch die „Verkleinerung der Produkte" umfasst. Hierin kann eine freundliche Umschreibung für die Unterlassung von gründlicher Prozessführung gesehen werden; ganz klar sind hier jedenfalls die oben beschriebenen beschleunigten und besonders beschleunigten Verfahren zu nennen, die Gesetzgeber und Finanz- und Justizministerien erdacht haben.

$\mathrm{Zu}$ konstatieren ist dabei, dass auch die Informalisierung in Gestalt von Diversion - in Deutschland Einstellungen von Strafverfahren durch die Staatsanwaltschaft nach $\S \S 153 \mathrm{ff}$. StPO - zu einem nicht geringen Teil deshalb so erfolgreich ist, weil sie zu einer Entlastung der Justiz insgesamt führt; auch sie kann daher zur Produktverkleinerung gezählt werden. Insofern hat sie, nachdem sie in den 1970er Jahren als Teil der „vier D“ der liberalen Kriminalpolitik (decriminalisation, deinstitutionalisation, diversion and due process, hierzu Lilly, Cullen \& Ball 2018: 150 ff.) galt, einen Niedergang erfahren: Sie wird heute kriminalpolitisch nicht von Entkriminalisierung und Rechtsstaatlichkeit begleitet, sondern zur Entlastung der Justiz instrumentalisiert. Sie birgt so das Risiko der ungleichen Rechtsanwendung (entsprechend Burghardt 2019: $269 \mathrm{f}$.): Diejenigen, die zwar Bagatelldelikte begangen haben, bei denen man aber unterstellt, man könne einen Bescheid nicht zustellen und die nach § 153a StPO verhängte Geldauflage nicht eintreiben, riskieren einen Strafprozess, aber eben ein „verkleinertes Produkt“. Andere, die von ihren Lebensumständen her eher dem Bild einer braven Bürgerin entsprechen, profitieren von der informalisierten Erledigung.

\section{Prozedurale Gerechtigkeit und Zugang zum Recht}

Die „Theorie der Prozeduralen Gerechtigkeit“ (Lind/Tyler 1988, Tyler 1990/2006 etc.) als sozialpsychologisches Gegenstück des Fairnessprinzips müsste an sich auch in Rechtskreisen auf breites Interesse stoßen; sie wird jedoch im deutschsprachigen Raum recht wenig diskutiert (vgl. aber Machura 2002, Köhnken 2019). Jedenfalls in der anglophonen Kriminologie hat sie für die Forschung zum Strafverfahren erhebliche Bedeutung und ist auch für das hier interessierende Thema wichtig: Es geht grundsätzlich um die Eignung des Verfahrens zur gerechten Verteilung von Ergebnissen. Dabei ist bedeutsam, dass im Verfahren nicht nur 
ein Ergebnis (Wahrheit/Recht - oder konkreter: Freispruch/Verurteilung bzw. eine bestimmte Sanktion) erbracht wird, sondern auch eine weitere Leistung, nämlich die Förderung von Akzeptanz der Entscheidung. Zur Feststellung dieser Eignung des Verfahrens wird die Perspektive der Rechtsunterworfenen bzw. die von einer Entscheidung Betroffenen eingenommen, für die das erlebte Verfahren eine erhebliche Bedeutung hat. Für die Kriminologie ist daher die Frage, warum Menschen sich an Gesetze halten (,Why people obey the law“, Tyler 1990/2006) eine fundamentale Ergänzung zur Frage, warum sie es nicht tun und straffällig werden. Die Procedural Justice-Forschung geht dabei grundsätzlich auch davon aus, dass dies zunächst auf ihren eigenen Überzeugungen und Werten gründet, die im Laufe der Sozialisation geprägt werden. Gesetze werden aber auch dann befolgt, wenn und weil sie für legitim gehalten werden. Legitimität wird dabei als Anerkennung staatlicher Gesetze, Autoritäten und deren Handlungen umschrieben, die vor allem dann vorhanden ist, wenn angenommen wird, dass das Rechtssystem und die handelnden Personen einen angemessenen Verhaltensstandard vorgeben, d.h. anerkennungswürdig sind. In einer großen Befragung Chicagoer Bürger Ende der 1980er Jahren ergab sich, dass die Beurteilung der Anerkennungswürdigkeit ganz entscheidend davon abhängt, ob ein erlebtes Verfahren der staatlichen Machtausübung als „fair“ betrachtet wird bzw. ob sich die Amtsträger fair verhalten haben. Die Anerkennung wiederum ist eine Voraussetzung für späteres normkonformes Verhalten. Da in zwei Befragungswellen auch selbstberichtete Delinquenz erhoben wurde, gelang es hier, für den Faktor der wahrgenommenen Legitimität der Strafverfolgungsbehörden eine eigenständige Bedeutung für die spätere compliance nachzuweisen, der z.B. den der Abschreckung überstieg (Tyler 2006: 63ff.). Wenn die wahrgenommene Fairness eines Strafverfahrens wichtig für die Normtreue sein kann, können sich dementsprechend Enttäuschungen in diesem Bereich negativ auswirken.

Daneben sollten auch andere ältere kriminologische Forschungsergebnisse zum Strafverfahren nicht in Vergessenheit geraten: Der enorm einflussreiche und prinzipiell strafrechts- und institutionenkritische Text des norwegischen Kriminologen Nils Christie „Conflicts as Property“ (1977) erinnert daran, dass es im Strafrecht zunächst um zwischenmenschliche Konflikte geht. Er enthält unter anderem Überlegungen dazu, wie der am Laien - d.h. an Opfern und Angeklagten - vorbei geführte Strafprozess, in dem letztlich nur Gericht, Staatsanwaltschaft und ggf. die Verteidigung wirken können, bei dem also der Konflikt deren professionellen Bedürfnissen untergeordnet wird, seine konfliktlösende Funktion verfehlt. Ebenfalls ein wichtiger Befund stammt von Feeley (1979), er ist ebenfalls schon dem Titel der Veröffentlichung zu entnehmen: „The process is the punishment“. Seine abschließende Hypothese ist, dass auch formalen Kriterien genügende Verfahren wegen ihrer Länge und der prozesssichernden 
Maßnahmen für die Angeklagten so belastend sind, dass sie alles daransetzen, die Verfahren abzukürzen, auch um den Preis des Verlusts von Rechtssicherheit.

Verstärkt greift sowohl die menschenrechtliche als auch die sozialwissenschaftliche Forschung derzeit den Gedanken des Zugangs zum Recht - access to justice - auf. Die Frage nach dem Zugang wird vielfach in der Außen-und Entwicklungspolitik gestellt (Kötter 2018), ist aber bei Weitem nicht ohne Belang für europäische (Straf-)Rechtssysteme. Untersucht werden z. B. die Rechtskenntnis (Bildung, Sprache) und Finanzierbarkeit der Rechtsverfolgung (Prozesskostenhilfe, Pflichtverteidigung) als Bedingungen der wirksamen Rechtsgewährung und seiner fundamentalen Bedeutung (Rudolf 2014, European Union Agency for Fundamental Rights and Council of Europe 2016). ${ }^{12}$ In diesem Zusammenhang ist auch die Kritik an einem normativ-prozeduralen bzw. auf das prozessuale beschränkte Verständnis der Menschenrechte zu nennen (Snacken 2015, Armstrong 2019, Morgenstern \& van Zyl Smit 2020). Insofern stellte sich im eingangs vorgestellten DETOUR-Projekt die Frage, ob das vielerorts zu beobachtende Bemühen, das Strafverfahren beschleunigt $\mathrm{zu}$ erledigen, in Haftsachen als materielle Verkürzung des Zugangs zum Recht angesehen werden kann. Dies ist dann der Fall, wenn mit weniger Skrupel Untersuchungshaft angeordnet wird - zur Beseitigung der Skrupel dient die Überlegung, dass dann ja immerhin die Dauer des Gesamtverfahrens verringert wird. Betroffen sind vor allem Beschuldigtengruppen, die als sozial Marginalisierte mangels festem Wohnsitz oder Drogenabhängigkeit eine Untersuchungshaft in den Augen der Justiz eher rechtfertigen bzw. leichter begründbar machen, auch wenn Bagatelldelikte Gegenstand des Verfahrens sind. Wird auf diese Weise unangemessen Untersuchungshaft verhängt, und sei sie nur kurz, leidet die Rechtsposition der Beschuldigten im oben beschriebenen Sinne doppelt, die grundsätzlich einer als unschuldig geltenden Person die gründliche Vorbereitung ihrer Verteidigung in Freiheit zubilligt.

\section{Untersuchungshaft und Untersuchungsgefangene: Stiefkinder der Justiz}

Diese Fragestellung erfordert eine Betrachtung des Kontextes der Untersuchungshaftverhängung. Wird über die Untersuchungshaft gesprochen und geschrieben,

$12 \mathrm{Zu}$ den festgestellten oder vermuteten Defiziten auf diesem Gebiet arbeitet seit 2018 ein europäisches Forschungsnetzwerk, https://www.accesstojust.net/ (abgerufen am 1.10.2019). 
finden sich nicht selten starke Worte - sie sei „Freiheitsberaubung gegenüber Unschuldigen“ (Hassemer 1984: 4), sie sei das „Stiefkind der Justiz“ (Reindl, Nickolai \& Gehl 1995). In der Tat ist sie das ungeliebte und vernachlässigte Aschenputtel in Strafprozessrecht und -praxis: Sie bedeutet Haft, ohne Strafe sein zu dürfen; sie ist ein Zwangsmittel, das einen nur schwer aufzulösenden Widerspruch zur Unschuldsvermutung mit sich bringt (Stuckenberg 1998: 530 ff., Morgenstern 2018: $516 \mathrm{ff}$.); ihre Voraussetzungen sind komplex; ihr Vollzug ist teuer. Von Verfassungs wegen muss sie das letzte Mittel der Prozesssicherung sein; falls sie angeordnet wird, muss sie - wie gesehen - möglichst kurz sein. Diese Gemengelage stellt hohe Anforderungen an die Praxis, denn Staatsanwaltschaft und Gerichte stehen unter Zeitdruck und unterliegen erheblichen Begründungszwängen (Morgenstern 2018: 488ff.). Zeitlicher Druck baut sich auch für die Verteidigung auf, die mitunter vor dem Dilemma steht, bei einem energischen Vorgehen gegen den Haftbefehl die Verteidigungsstrategie zu gefährden und das Verfahren insgesamt zu verzögern (Schlothauer, Weider \& Nobis 2016: $434 \mathrm{ff}$;; auch Hammerschick \& Reidinger 2016: 72 ff. für Österreich).

Ein klassischer Vorwurf lautet, in Deutschland werde „zu oft, zu schnell, zu lang“ Untersuchungshaft angeordnet (z. B. Arbeitskreis Strafprozessreform 1983: 25, Deutscher Anwaltverein 2007: 4). Für eine ganze Weile schien dieser Vorwurf er jedenfalls in seiner Schärfe seine Berechtigung verloren zu haben, denn zwischen 1994 und 2013 nahmen die stichtagsbezogene Zahl der Untersuchungsgefangenen kontinuierlich ab und halbierte sich fast (Abbildung 1).

Nicht nur der historische Vergleich, auch der Ländervergleich lässt Deutschland mit einer der niedrigsten Untersuchungsgefangenenraten in Europa gut aussehen. 14 Untersuchungsgefangene pro 100.000 Einwohner waren es im Jahr 2013, 16 im Jahr 2016; Frankreich oder die Niederlande hatten fast doppelt so viele, auch der europäische Durchschnitt liegt weit höher (Morgenstern 2018: $73 \mathrm{ff}$.).

Die Gründe für die über lange Zeit günstige Entwicklung sind nicht klar zu benennen, die Diskussion soll hier nicht aufgegriffen werden (zur Rolle der Verteidigung Morgenstern 2019: 475 ff., zur Dauer des Verfahrens Dessecker 2007). Hingewiesen werden soll aber auf ein weiteres Charakteristikum der deutschen Untersuchungshaftpraxis: Beschuldigte und Angeklagte ohne deutschen Pass sind weit überrepräsentiert. Dies ist vor allem durch den Haftgrund der Fluchtgefahr bedingt, der für Menschen ohne festen Wohnsitz in Deutschland oder mit etablierten Kontakten ins Ausland oft routinemäßig angenommen wird. Diese Daten werden statistisch nicht gesondert ausgewiesen, die hier in Tabelle 1 verwendeten Daten stammen aus einer eigenen Erhebung aus dem Jahr 2013 - es spricht einiges dafür, dass die Anteile jetzt noch höher ausfallen würden. Ähnlich wie in der Schweiz und auch in Österreich sind jedenfalls in den Stadtstaaten und 


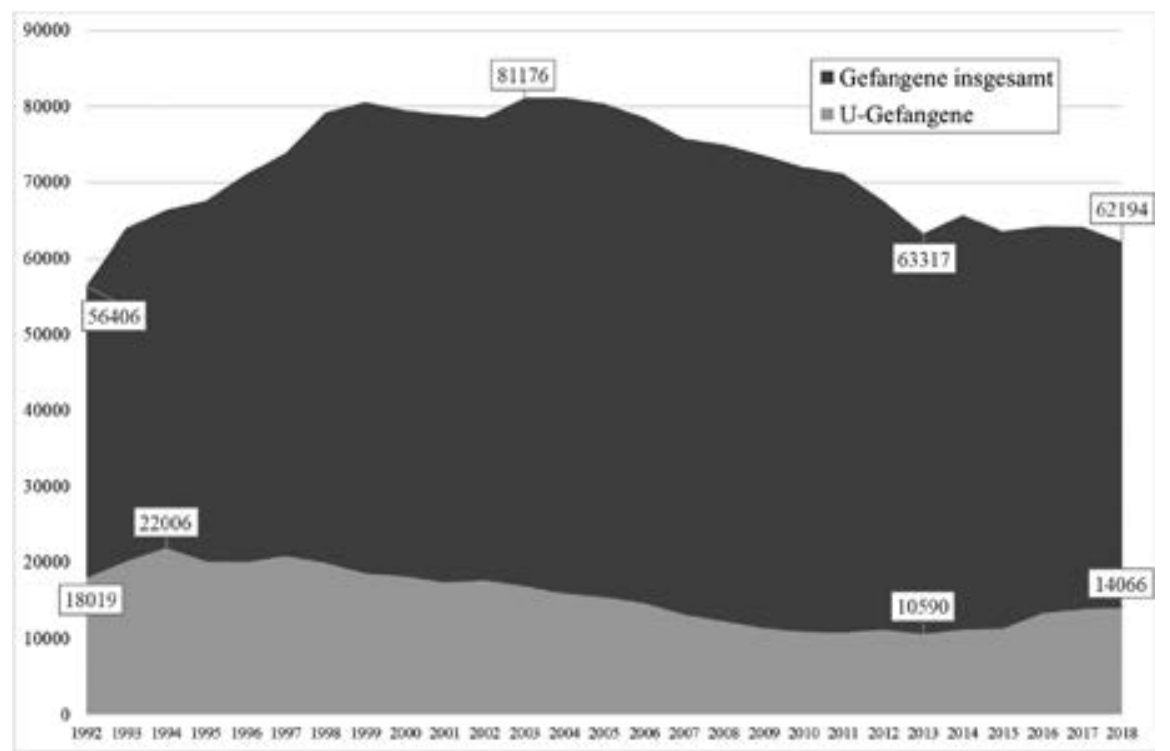

Abbildung 1: Entwicklung der Gefangenenzahlen in Deutschland 1992-2018

Quelle: Statistisches Bundesamt 2018 (Strafvollzugsstatistik: Bestand der Gefangenen und Verwahrten) und früher. Stichtag: 31.3. (2001: 31.1. wegen des Umzugs des Bundesjustizministeriums nach Bonn).

einigen der Flächenländer weit über die Hälfte der U-Gefangenen Ausländer, das liegt deutlich über ihrem Anteil an der Bevölkerung, an den Tatverdächtigen, den Verurteilten und vor allem auch an den Strafgefangenen (ausführlich Morgenstern 2018: $396 \mathrm{ff}$.).

Eine besorgniserregende Entwicklung zeigt sich in den letzten Jahren, denn wie aus Abbildung 1 ersichtlich hat die günstige Tendenz nicht angehalten. Eine stichtagbezogene Zunahme von knapp 3500 zwischen 2013 und 2018, die keine Entsprechung bei der Zahl der Strafgefangenen hat, bedeutet eine Zunahme von fast einem Drittel binnen fünf Jahren. Diese Entwicklung bringt in manchen Regionen eine Wiederkehr zumindest zeitweise überbelegter Vollzugsanstalten, wie aus Medienberichten und Anfragen in den Länderparlamenten hervorgeht. ${ }^{13}$

13 Z. B. Hamburger Bürgerschaft, Drucksache 21/9059 vom 19.5.2017; Landtag RheinlandPfalz, Drucksache 17/1884 vom 19.12.2017; zur Situation in Stuttgart https://www.suedkurier. de/ueberregional/panorama/Ich-weiss-nicht-wo-ich-die-Leute-noch-unterbringen-soll-VieleGefaengnisse-in-Deutschland-leiden-unter-UEberbelegung;art409965,10006512 (abgerufen am 1.10.2019). Statistisch spiegelt sich dies wegen der länderbezogenen Durchschnittswerte in der Statistik des Statistischen Bundesamte zum „Bestand der Gefangenen und Verwahrten in den 
Auch hier liegen noch keine genauen Analysen vor, es spricht aber viel dafür, dass es weniger die Dauer von Strafverfahren als die steigende Anzahl von Inhaftierungen bei ausländischen Tatverdächtigen ist, die die Zahlen der Untersuchungsgefangenen insgesamt haben steigen lassen - dies ergibt sich recht klar etwa für Hamburg (Statistisches Bundesamt 2019; Heinzle, Salzwedel \& Spanner 2019).

Tabelle 1: Ausländische Untersuchungsgefangene 2013 nach Bundesländern

\begin{tabular}{|c|c|c|c|c|c|}
\hline & $\begin{array}{l}\text { Ausländer } \\
\text { in \% aller } \\
\text { U-Gefangenen }\end{array}$ & $\begin{array}{l}\text { EU-Ausländer } \\
\text { in \% aller } \\
\text { U-Gefangenen }\end{array}$ & & $\begin{array}{l}\text { Ausländer } \\
\text { in \% aller } \\
\text { U-Gefangenen }\end{array}$ & $\begin{array}{l}\text { EU-Ausländer } \\
\text { in \% aller } \\
\text { U-Gefangenen }\end{array}$ \\
\hline $\begin{array}{l}\text { Baden- } \\
\text { Württemberg }\end{array}$ & $59 \%$ & $27 \%$ & $\begin{array}{l}\text { Nordrhein- } \\
\text { Westfalen }\end{array}$ & $56 \%$ & $26 \%$ \\
\hline Bayern & $55 \%$ & $32 \%$ & Niedersachsen & $40 \%$ & $19 \%$ \\
\hline Berlin & $67 \%$ & $29 \%$ & $\begin{array}{l}\text { Rheinland- } \\
\text { Pfalz }\end{array}$ & $44 \%$ & $19 \%$ \\
\hline Brandenburg & $39 \%$ & $36 \%$ & Saarland & $53 \%$ & $32 \%$ \\
\hline Bremen & $34 \%$ & $14 \%$ & Sachsen & $31 \%$ & $15 \%$ \\
\hline Hamburg & $76 \%$ & $33 \%$ & $\begin{array}{l}\text { Sachsen- } \\
\text { Anhalt }\end{array}$ & $20 \%$ & $10 \%$ \\
\hline Hessen & $55 \%$ & $22 \%$ & $\begin{array}{l}\text { Schleswig- } \\
\text { Holstein }\end{array}$ & $33 \%$ & $11 \%$ \\
\hline \multirow[t]{2}{*}{$\begin{array}{l}\text { Mecklenburg- } \\
\text { Vorpommern }\end{array}$} & $28 \%$ & $21 \%$ & Thüringen & $20 \%$ & $15 \%$ \\
\hline & & & $\begin{array}{l}\text { Total (16 Bun- } \\
\text { desländer) }\end{array}$ & $53 \%$ & $26 \%$ \\
\hline
\end{tabular}

Quelle: Morgenstern 2018: 411.

deutschen Justizvollzugsanstalten nach ihrer Unterbringung auf Haftplätzen des geschlossenen und offenen Vollzugs jeweils zu den Stichtagen 31. März, 31. August und 30. November eines Jahres“, die auch die in der Regel nicht voll belegten Plätze des offenen Vollzugs einrechnen, aber (noch) nicht wider. 


\section{Erkenntnisse aus dem DETOUR-Projekt}

\section{Forschungsfragen und -methoden}

Vor diesem Hintergrund erschien aus deutscher Sicht eine vergleichende Studie interessant, die die Bewältigungsstrategien der geschilderten vielfältigen Probleme in der nationalen Untersuchungshaftpraxis in den Blick nahm. Entsprechend hatte die Studie „Detour - Towards Pre-trial Detention as Ultima Ratio“ die Haftund Haftvermeidungspraxis in sieben europäischen Staaten (neben Deutschland auch Belgien, Irland, Litauen, die Niederlande, Österreich und Rumänien) zum Gegenstand, der Abschlussbericht wurde 2018 vorgelegt. ${ }^{14}$ Der Ausgangspunkt ist die auf die Menschenrechte der EMRK und das nationale Verfassungsrecht gegründete Annahme, wegen der Bedeutung des Freiheitsrechts könne Untersuchungshaft nur als letztes Mittel gerechtfertigt werden. Schwerpunkte der Betrachtung waren die Legitimität der Haftgründe und der Entscheidungsprozeduren sowie die Verfügbarkeit von alternativen Prozesssicherungsmechanismen. Neben einer Analyse der rechtlichen Rahmenbedingungen, der Rechtsprechung und dem Forschungsstand in den sieben Teilnehmerstaaten, die in entsprechenden Länderberichten zusammengefasst wurden, wurden im empirischen Teil der Studie zunächst explorativ Haftverhandlungen beobachtet und einige Akten ausgewertet. Mit einem aus diesen Materialien und Beobachtungen entwickelten einheitlichen Interviewleitfaden wurden pro Partnerland dann rund 30 Praktikerinnen und Praktiker aus Staatsanwaltschaften, Gerichten, der Anwaltschaft und der Vollzugspraxis zu ihren Erfahrungen und Werthaltungen befragt. Diese Erkenntnisse wurden länderspezifisch thematisch analysiert und schließlich einer vergleichenden Betrachtung unterzogen. Die deutschen Interviews fanden überwiegend in Berlin, einige in Mecklenburg-Vorpommern, Hamburg und Nordrhein-Westfahlen statt (zur Methodik näher Morgenstern 2017: 77 ff.).

\section{Temporale Aspekte: Untersuchungshaft, aber kurz!}

Geht man von der zentralen Fragestellung im DETOUR-Projekt aus, so ist die Vermeidung von Untersuchungshaft per se etwas Gutes, etwas, das anzustreben ist. Idealerweise wird Untersuchungshaft gar nicht angeordnet, passiert es doch, sind die Betroffenen bestrebt, sie möglichst schnell enden zu lassen. Die Justiz hat daneben aber noch andere Bestrebungen, die sich aus dem geschilderten theo-

14 Alle Ergebnisse sind abrufbar unter www.detour.at/irks. 
retischen Rechtsrahmen und den praktischen Effizienzbestrebungen ergeben: Sie will das Verfahren an sich möglichst zügig beenden; dazu braucht sie die angeklagte Person ,griffbereit“ und Haftprüfungen verzögern das Verfahren. Und von Verteidigungsseite steht ebenfalls auch nicht immer die Beendigung der Untersuchungshaft, sondern eine glimpfliche Beendigung des ganzen Verfahrens im Vordergrund; auf das Dilemma, das der Kampf gegen den Haftbefehl oft nicht mit einer Verteidigungsstrategie zusammengeht, ist bereits hingewiesen worden.

Die Praxis erkennt die Eilbedürftigkeit von Haftsachen grundsätzlich an und versucht sie durch verschiedene Vorkehrungen auch umzusetzen - in Deutschland wird dies z. B. symbolisiert durch den Aufkleber „Haft“! auf den Akten oder den pinkfarbenen Haftbefehl, der sich in der Akte sofort findet. Oft wurde uns erklärt, man sei sich des Zeitablaufs immer bewusst; geschildert wurde, welche verfahrenstechnische Vorkehrungen für einen zügigen Ablauf getroffen sind z. B. bei der Staatsanwaltschaft die sofortige Anlegung von Doppelakten in Haftsachen. Oft wurde auch beschrieben, woran es bei den Abläufen noch mangelt (z. B. elektronische Aktenführung) was zumindest für ein entsprechendes Problembewusstsein spricht. Solche Befunde finden sich in allen Länderhebungen (z. B. für Belgien [Wallonie] Jonkheere 2017: 2, für Österreich Hammerschick \& Reidinger 2017: 74; für Deutschland Morgenstern 2017: 95 f., für Litauen mit Blick auf die dort eingeführte elektronische Akte Bikelis \& Pajaujis 2017: 37; zusammenfassend Hammerschick et al. 2018: $51 \mathrm{ff}$.).

Besonders in der deutschen Teilstudie war aber auffällig, dass es einen Typus von Fällen gibt, den alle Justizbeteiligten gerne ,irgendwie“ und, wenn es denn geht, schnell und zur Gänze erledigt haben wollen. Dafür sind sie auch bereit eine begrenzte Untersuchungshaftzeit in Kauf zu nehmen: Das Eingangszitat „was anderes fällt mir da nicht ein“ - das aus einem sehr differenzierten Interview stammt, das keineswegs von Hardlinern bestritten wurde - ist ein solcher Beleg; ein weiteres typisches Zitat stammt aus dem Haftvermeidungsprojekt einer Justizvollzugsanstalt, noch eines von einem anderen Gericht:

SozialarbeiterIn JVA, Projekt Haftvermeidung, Interview 25: „Inzwischen haben wir auch viele beschleunigte Verfahren mit Hauptverhandlungshaft [Anm. CM: max. eine Woche], da haben wir dann nichts mit zu tun, das finde ich gut, denn es reduziert die Untersuchungshaft, z. B. bei kleinen Vermögensdelikten.“

RichterIn, Interview 17: "Was wir bei ausländischen Tätern machen ist ein kurzer Prozess. Also Untersuchungshaft dauert zwei Wochen, dann halt ein beschleunigtes Verfahren.“

Auch Verteidiger drücken aufs Tempo, ohne immer gegen die Haft an sich anzugehen, auch hier geht es um Kosten-Nutzen-Erwägungen - manchmal für die eigene Arbeit, in diesem Beispiel hier aber für das Gesamtergebnis für den Mandanten: 
VerteidigerIn, Interview 5: „Was weiß ich, Diebstähle oder sonst was, aber keinen festen Wohnsitz, die kommen in Haft. Da bin ich ganz schnell bei der Staatsanwaltschaft, bei solchen einfachen Fällen, auf frischer Tat ertappt, will ich gar keine Akteneinsicht haben, sondern will vom Staatsanwalt nur, dass der ganz schnell anklagt, [...] Das ist dann die einzige und effektivste Art die U-Haft gering zu halten. Es ist völlig klar, dass die rauskommen, da gibt‘s keine Bewährung oder so was, sondern wirklich nur einkassiert, weil keinen festen Wohnsitz.“

\section{Vergleichende Erkenntnisse}

Beschleunigte bzw. vereinfachte Verfahren sind mancherorts noch weit populärer als in Deutschland, in unserer Untersuchungsgruppe traf dies auf die Niederlande, Belgien und Österreich zu; in allen drei Staaten gibt es relativ viele Untersuchungsgefangene und auch der Anteil von Ausländern ist hoch (im Überblick Morgenstern 2018: $71 \mathrm{ff}$.). Auch in Litauen wird von der Praxis ein beschleunigtes Verfahren geschätzt, das jedoch vor allem die wenigen ausländischen Tatverdächtigen in Bagatellverfahren trifft (Bikelis \& Pajaujis 2017: 9). Irland ist insofern eine Ausnahme, als dort Untersuchungshaft eine untergeordnete Rolle spielt; stattdessen wird regelmäßig conditional bail angeordnet, so dass die beschuldigte Person während des Verfahrens unter Auflagen auf freiem Fuß bleibt. Auch sind die zeitlichen Vorgaben für die Abwicklung eines Strafverfahrens unabhängig von der Frage einer Haft deutlich straffer als z. B. in Deutschland (Hammerschick et al. 2018: 50 f. mit Fristen im Ländervergleich). Dennoch waren auch die irischen Befragten oft der Auffassung, dass Verfahren nicht zügig genug geführt würden und dies zu Lasten von denjenigen, die doch einmal in Untersuchungshaft genommen würden, gehe (Perry \& Rogan 2017: 31f.).

Die Beschleunigungsdiskussion wird lebhaft in den Niederlanden geführt. Hier gibt es zwei Spielarten beschleunigter Verfahren (sog. ZSM-Verfahren: (' $z o$ snel mogelijk')), bei denen der Polizei eine Filterfunktion zukommt und denen wenigsten teilweise Haftvermeidungspotenzial zugesprochen wird (Boone, Jacobs \& Lindemann 2017: 29 ff.). Auch sie werden mit Blick auf die Gewährung rechtlichen Gehörs bzw. die Verfahrensfairness angezweifelt (Jacobs \& van Kampen 2014); wurden aber in der niederländischen Teilstudie von Interviewten aller drei Professionen auch als gut handhabbar und entlastend beschrieben. In Österreich wird der Beschleunigungsgedanke ebenfalls stark betont, hier soll vor allem mit der Figur des „direkten Strafantrags“, der von der Staatsanwaltschaft mit dem Antrag auf Untersuchungshaft zu koppeln ist, eine schnellere Anklageerhebung (in der Regel nach sechs Wochen) erreicht werden. Hier gab es vor allem von Seiten der Gerichte viel Skepsis in Bezug auf die Gründlichkeit der Vorbereitung 
einer solchen Anklage (Hammerschick \& Reidinger 2017: 74 ff.). Aus Belgien kommt der interessante Befund, dass Untersuchungshaft für manche Verteidiger deshalb akzeptabel ist, weil die Verfahren wegen des Beschleunigungsgebotes etwa doppelt so schnell abgeschlossen werden wie andere. Diese Akzeptanz gibt es jedenfalls, wenn ohnehin eine Freiheitsstrafe zu erwarten ist (für die Wallonie Jonckheere 2017: 37). Zu Recht wird zu der auch in Österreich vereinzelt geäußerten Ansicht aber darauf hingewiesen, dass die Freiheitsstrafe das Resultat einer self-fulfilling prophecy werden kann und bislang von Verteidigungsseite eher galt, dass die Chancen auf eine bedingte, d.h. zur Bewährung ausgesetzte Freiheitsstrafe größer sind, wenn der Mandant oder die Mandantin das Urteil auf freiem Fuß entgegennehmen kann (Hammerschick \& Reidinger 2017: 76).

\section{Die Marginalisierten des Rechts - Für wen wollen wir uns ein gerechtes Verfahren leisten?}

Das Dargelegte zeigt stabil wirkende menschen- und verfassungsrechtliche Grundlagen, die als beschuldigtenschützend zu verstehen sind - in Art. 6 EMRK ist nicht von einem schnellen oder gar beschleunigten Verfahren die Rede, sondern von einem „Verfahren angemessener Dauer“. Bei der Frage der Angemessenheit hat der EGMR deutlich gemacht, dass „die besondere Beschleunigung, auf die der Inhaftierte während der Prüfung seines Falles ein Recht hat, nicht der Sorgfalt schaden darf, mit der die Ermittlungsbehörden ihren Aufgaben nachkommen. “15 In der deutschen, aber auch den benachbarten Rechtsordnungen zeigt sich demgegenüber, dass einfachgesetzliche Bestimmungen und die Justizpraxis bei den Beschleunigungsbemühungen weniger die beschuldigtenschützende als vielmehr die entlastende Wirkung im Auge haben.

Die Aufmerksamkeit von Politik, Literatur und Berufsverbänden gilt vornehmlich den schwierigen Fällen vor den Landgerichten, d.h. den Umfangsverfahren, oft dem Wirtschaftsstrafrecht. Die Justizpraxis beklagt eine Überlast, allerorten wird versucht, die vorhandenen Kapazitäten zu strecken und möglichst viel zu erledigen. Darüber hinaus wird die Aufgabe, gerade Haftsachen möglichst zügig $\mathrm{zu}$ erledigen, oft als besondere Bürde wahrgenommen, wegen derer man sich dann eben weniger um andere Sachen kümmern kann. Das führt dazu, dass Alltagsfälle vor den Amtsgerichten rechtsstaatlich marginalisiert zu werden drohen,

15 Z. B. EGMR, Urt. v. 13.2.2001 - $34947 / 97$ (Richet ./. Frankreich), para. 66 (meine Übers.); vgl. auch EGMR, Urt. v. 9.7.2015 - 8824/09 and 42836/12 (El Khoury ./. Deutschland), para. 58. 
vor allem, wenn sie Randgruppen betreffen. Wenngleich es inzwischen immerhin den - ausdrücklich auch mit Ressourcenproblemen begründeten - Versuch gibt, das Schwarzfahren zu entkriminalisieren (Gesetzesantrag des Freistaats Thüringen, Bundesratsdrucksache 424/19 vom 10.9.2019) ist die Entlastung der Strafjustiz durch Entkriminalisierung für die Politik keine ernsthaft diskutierte Option.

Die vergleichenden Befunde zeigen, dass die Beschleunigungsfreude nicht nur in Deutschland groß ist; spezielle beschleunigte Verfahren oder jedenfalls ein beschleunigtes Vorgehen werden für bestimmte Tätergruppen auch als kleineres Übel und Mittel der Vermeidung langer Untersuchungshaft akzeptiert. Dahinter steckt oft Effizienzdenken in Bezug auf die eigene Arbeit, sowohl bei Staatsanwaltschaften und Gerichten als auch von Seiten der Verteidigung. In unseren Studienergebnissen finden sich zahlreiche Hinweise darauf, dass die Beschleunigung des Verfahrens als Wert an sich verstanden wird, der andere, die beschuldigtenschützende Überlegungen zurücktreten lässt und allen befragten Berufsgruppen zur Rechtfertigung dient; beispielsweise bei der Frage, warum Verhältnismäßigkeitsüberlegungen in Bezug auf die Haftanordnung auch bei Bagatelldelikten, die ausländischen, reisenden und wohnsitzlosen Beschuldigten als typischen „Marginalisierten des Rechts“ (Lubitz 2019: 282) zu Last gelegt werden, nicht durchgreifen - immerhin, so der Tenor, geht es dann aber schnell.

Es spricht daher einiges dafür, dass die derzeitige Praxis, insbesondere dann, wenn sie die geschilderten Expressverfahren nutzt, die erforderliche prozessuale Fairness vernachlässigt: Wie kann jemand, der „am Fließband“ verurteilt wird, den Prozess als legitim erleben? Diese der Ökonomisierung der Justiz zuzuordnenden Beschleunigungsbemühungen sind außerdem selbst mit Blick auf ihre Effizienz - und aus Sicht der DETOUR-Forschung auf ihr Haftvermeidungspotenzial - fragwürdig: Die Entlastung des Justizsystems als Ganzem geht dann verloren, wenn eine Geldstrafe ohne sinnvolle Ermittlungen zur Leistungsfähigkeit verhängt wird und ohnehin mit einer auf einem teuren Haftplatz vollzogenen Ersatzfreiheitsstrafe endet; dasselbe gilt für Widerrufe bei bedingten Freiheitsstrafen, die ohne die notwendigen Ermittlungen zur sozialen Situation und ohne Begleitmaßnahmen verhängt werden. Bedenkenswert ist auch der Vorwurf, diese Beschleunigungstendenzen führten zu einem Sonderstrafrecht für unverteidigte Wohnsitzlose und ausländische Beschuldigte; zu einem Befund des „punishing the poor" ist es dann nicht mehr weit.

Kriminalpolitisch ist den immer weitergehenden Forderungen nach Beschleunigung entgegenzuhalten, dass ein Kriminalsystem sich einen ordentlichen Prozess leisten muss, wenn es glaubwürdig - legitim! - bleiben will. Wenn der betreffenden Rechtsordnung die prozessordnungsgemäße und rechtsstaatliche Aburteilung wichtig ist, muss sie die Ressourcen aufstocken oder sie muss ohne Preisgabe ihrer Rechtsstaatlichkeit die Justiz von Bagatellen entlasten. Das ist 
letztlich nur durch Entkriminalisierung von Bagatellen (z. B. Schwarzfahren, Bagatelldiebstähle bis zu einem gewissen Wert) möglich - entsprechende Taten könnten dann weder in Untersuchungshaft noch in einem für die Justiz offenbar lästigen Prozess resultieren. Welche Effekte „marginalisierte Strafverfahren“ auf die betroffenen Justizprofessionellen und schon gar auf die „Nutzer“, d.h. die von Gerichtsverfahren als Angeklagte, Opfer, Zeuginnen oder Zeugen tatsächlich betroffenen Bürgerinnen und Bürger haben, sollte zudem verstärkt auch Gegenstand der kriminologischen und rechtssoziologischen Justizforschung sein.

\section{Literaturverzeichnis}

Arbeitskreis Strafprozessreform (1983) Die Untersuchungshaft. Gesetzentwurf mit Begründung. Heidelberg: Müller.

Armstrong, Sarah (2018): Securing Prison through Human Rights: Unanticipated Implications of Rights-Based Penal Governance. The Howard Journal of Crime and Justice 57: 401-421.

Bottoms, Anthony (1995) The philosophy and politics of punishment and sentencing, S. 7-49 in C. Clarkson \& R. Morgan (Hrsg.), The Politics of Sentencing Reform. Oxford: Clarendon Press.

Burghardt, Boris (2019) Informalisierung des Rechts, S. 259-274 in C. Boulanger, J. Rosenstock \& T. Singelnstein (Hrsg.), Interdisziplinäre Rechtsforschung. Wiesbaden: Springer.

Christie, Nils (1977) Conflicts as Property. British Journal of Criminology 17: 1-15.

Baumanns, Silke (2011) Der Beschleunigungsgrundsatz im Strafverfahren: die Verfahrensüberlänge und ihre Rechtsfolgen. Baden-Baden: Nomos.

Beulke, Werner \& Swoboda, Sabine (2018): Strafprozessrecht. 14. Aufl., Heidelberg: C. F. Müller.

Bikelis, Skirmantas \& Pajaujis, Virgilijus (2017): DETOUR - Towards Pre-trial Detention as Ultima Ratio. 2nd Lithuanian National Report on Expert Interviews. Wien: http://www.irks. at/detour/publications.html (abgerufen am 1.10.2020).

Boone, Miranda, Jacobs, Pauline \& Lindemann, Joep (2017): DETOUR - Towards Pre-trial Detention as Ultima Ratio. 2nd Dutch National Report on Expert Interviews. Wien: http:// www.irks.at/detour/publications.html (abgerufen am 1.10.2020).

Bundesministerium der Justiz und für Verbraucherschutz (Hrsg.) (2015) Bericht der Expertenkommission zur effektiveren und praxistauglicheren Ausgestaltung des allgemeinen Strafverfahrens und des jugendgerichtlichen Verfahrens. Berlin: BMJV.

Deutscher Anwaltverein (2007) Stellungnahme des Deutschen Anwaltvereins durch den Strafrechtsausschuss zum Vorschlag für einen Rahmenbeschluss über die Europäische Überwachungsanordnung in Ermittlungsverfahren innerhalb der Europäischen Union KOM (2006) 468 endgültig. (www.anwaltverein.de, unter Interessenvertretung/ Stellungnahmen/Archiv, abgerufen am 1.10.2019)

Dessecker, Axel (2007): Strategien zur Vermeidung langer Untersuchungshaft, S. 269-278 in: F. Lösel, D. Bender \& J.-M. Jehle, (Hrsg.), Kriminologie und wissensbasierte Kriminalpolitik: Entwicklungs- und Evaluationsforschung. Mönchengladbach: Forum.

Ernst, Marcel (2001) Das beschleunigte Verfahren im Strafprozeß und seine Handhabung in Bochum. Aachen: Shaker. 
European Union Agency for Fundamental Rights and Council of Europe (2016) Handbook on European law relating to access to justice. Luxembourg: Publications Office of the European Union.

Feeley, Malcom (1979) The Process Is the Punishment: Handling Cases in a Lower Criminal Court. New York: Russell Sage.

Francioni, Francesco (Hrsg.) (2007) Access to Justice as a Human Right, New York: Oxford University Press.

Garland, David (1996) The limits of the sovereign state: strategies of crime control in contemporary society. British Journal of Criminology 36: 445-471.

Garland, David (2001) The Culture of Control: Crime and Social Order in Contemporary Society. Chicago: University of Chicago Press.

Hammerschick, Walter \& Reidinger, Viktoria (2017): DETOUR - Towards Pre-trial Detention as Ultima Ratio. 2nd Austrian National Report on Expert Interviews. Wien: http://www.irks.at/ detour/publications.html (abgerufen am 1.10.2020).

Hammerschick, Walter, Morgenstern, Christine, Bikelis, Skirmantas, Boone, Miranda, Durnescu, Ioan, Jonckheere, Alexia, Lindeman, Joep, Maes, Eric \& Rogan, Mary (2018): DETOUR Towards Pre-trial Detention as Ultima Ratio. 2nd Austrian National Report on Expert Interviews. Wien: http://www.irks.at/detour/publications.html (abgerufen am 1.10.2020).

Hassemer, Winfried (1984) Die Voraussetzungen der Untersuchungshaft. Strafverteidiger 4: 37-45.

Heinz, Wolfgang (2017) Kriminalität und Kriminalitätskontrolle in Deutschland - Berichtsstand 2015 im Überblick. Internet-Publikation: Konstanzer Inventar Sanktionsforschung <www. ki.uni-konstanz.de/kis/>, Version 1/2017.

Heinzle, Christoph, Salzwedel, Carl-Georg \& Spanner, Elke (2019) Deutlich mehr Menschen in U-Haft, Internetpublikation des Norddeutschen Rundfunks vom 24.4.2019 (https://www. ndr.de/nachrichten/hamburg/Deutlich-mehr-Menschen-in-U-Haft, untersuchungshaft102. html, abgerufen am 1.10.2019).

Jacobs, Pauline \& Van Kampen, Petra (2014) Dutch “ZSM Settlements" in the Face of Procedural Justice: The Sooner the Better? Utrecht Law Review 4: 73-85.

Jonckheere, Alexia (2017) DETOUR - Towards Pre-trial Detention as Ultima Ratio. Report on (French-speaking) Expert Interviews. Wien: http://www.irks.at/detour/publications.html (abgerufen am 1.10.2019). Prozedurale

Köhnken, Günter (2019) Gerechtigkeit und Respekt in der Justiz, S. 53-59 in I. Goeckenjan, J. Puschke \& T. Singelnstein (Hrsg.), Für die Sache - Kriminalwissenschaften aus unabhängiger Perspektive. Festschrift für Ulrich Eisenberg zum 80. Geburtstag. Berlin: Duncker \& Humblot: 53-59.

Kötter, Matthias (2017) Besserer Zugang zum Recht (Access to Justice) durch staatliche Anerkennung informeller Justizsysteme? Zur Relevanz rechtssoziologischer Forschung für die Außen- und Entwicklungspolitik, SFB-Governance Working Paper Series Nr. 74, Berlin: DFG Sonderforschungsbereich 700.

Krehl, Christoph \& Eidam, Lutz (2006) Die überlange Dauer von Strafverfahren. Neue Zeitschrift für Strafrecht 26: 1-10.

Kühne, Hans-Heiner (2010) Die realen und potentiellen Auswirkungen des Beschleunigungsgebots für die Struktur des Strafverfahrensrechts. Juristenzeitung 65: 821-828.

Leitch, Jennifer A. (2013) Looking for quality: the empirical debate in access to justice research. Windsor Yearbook of Access to Justice, 31: 229-55. 
Lilly, J. Robert, Cullen, Francis \& Ball, Richard A. (2018) Criminological Theory: Context and Consequences. 7. Aufl., London: Sage.

Lind, E. Allan \& Tyler, Tom, R. (1988). The social psychology of procedural justice. New York: Plenum.

Lubitz, Tobias (2010) Das beschleunigte Verfahren der StPO und seine rechtstatsächliche Durchführung in Berlin und Brandenburg. Berlin: Duncker \& Humblot.

Lubitz, Tobias (2019) Die Marginalisierten des Rechts - für einen Perspektivwechsel in der strafprozessualen Gesetzgebung. Neue Kriminalpolitik 31: 282-300.

Machura, Stefan (2001): Fairneß und Legitimität. Baden-Baden: Nomos.

Machura, Stefan (2002): Verfahrensgerechtigkeit aus der Perspektive von Beschuldigten, S. 195-232 in S. Machura \& S. Barton (Hrsg.), Verfahrensgerechtigkeit und Zeugenbeweis. Baden-Baden: Nomos.

Morgenstern, Christine (2017) DETOUR - Towards Pre-trial Detention as Ultima Ratio. 2nd German National Report on Expert Interviews. Wien: http://www.irks.at/detour/ publications.html (abgerufen am 1.10.2020).

Morgenstern, Christine (2018) Die Untersuchungshaft. Baden-Baden: Nomos.

Morgenstern, Christine (2019) Verteidigung bei Untersuchungshaft, S. 475-488 in I. Goeckenjan, J. Puschke \& T. Singelnstein (Hrsg.), Für die Sache - Kriminalwissenschaften aus unabhängiger Perspektive. Festschrift für Ulrich Eisenberg zum 80. Geburtstag. Berlin: Duncker \& Humblot.

Morgenstern, Christine \& van Zyl Smit, Dirk (2020) Internationale Menschenrechte im Justizvollzug: Wo stehen wir im Jahr 2020?, S. 861-931 in K. Drenkhahn, B. Geng, J. Grzywa, S. Harrendorf, C. Morgenstern \& I. Pruin (Hrsg.), Festschrift für Frieder Dünkel zum 70. Geburtstag. Mönchengladbach: Forum Verlag.

Paeffgen, Ulrich (1986): Vorüberlegungen zu einer Dogmatik des Untersuchungshaft-Rechts. Köln u. a.: Heymanns.

Perry, David \& Rogan, Mary (2017): DETOUR - Towards Pre-trial Detention as Ultima Ratio. Second National Report - Expert Interviews in Ireland. Wien: http://www.irks.at/detour/ publications.html (abgerufen am 1.10.2020).

Pest, Robert (2017) Das Verzögerungsverbot im Strafverfahren. Tübingen: Mohr Siebeck.

Putzke, Holm (2004): Beschleunigtes Verfahren bei Heranwachsenden. Holzkirchen: Felix-Verlag.

Reindl, Richard, Nickolai, Gerhard \& Gehl, Günter (Hrsg.) 1995: Untersuchungshaft, Stiefkind der Justiz. Weimar: Dadder.

Robinson, Gwen (2017) Stand-down and deliver: Pre-Sentence Reports, quality and the new culture of speed. Probation Journal 64: 337-353.

Röhl, Klaus Friedrich. (2009) Ökonomisierung der Justiz und richterliche Unabhängigkeit, S. 103-118 in Sächsisches Staatsministerium der Justiz und für Europa (Hrsg.), Impulse für eine moderne und leistungsstarke Justiz. Dokumentation Symposium Justizlehre Dresden 2009. Stuttgart: Boorberg.

Rudolf, Beate (2014) Rechte haben, Recht bekommen: Das Menschenrecht auf Zugang zum Recht. Berlin: Deutsches Institut für Menschenrechte.

Scheffler, Uwe (2014) Das beschleunigte Verfahren als ein Akt angewandter Kriminalpolitik, S. 437-448 in E. Graul und G Wolf (Hrsg.), Gedächtsnisschrift für Dieter Meurer. Berlin: de Gruyter.

Schlothauer, Reinhold, Weider, Hans-Joachim \& Nobis, Frank (2016) Untersuchungshaft. 5. Aufl., C. F. Müller: Heidelberg. 
Schröer, Eike (1998): Das beschleunigte Strafverfahren gem. §§ 417 ff. StPO. Berlin: Duncker \& Humblot.

Schulte-Kellinghaus, Thomas (2006) Die Ressourcengarantie für die Dritte Gewalt Verfassungsrechtliche Forderungen zur Gewährleistung einer unabhängigen Rechtsprechung. Zeitschrift für Rechtspolitik 39:169-172.

Singelnstein, Tobias \& Derin, Benjamin (2017) Das Gesetz zur effektiveren und praxistauglicheren Ausgestaltung des Strafverfahrens. Neue Juristische Wochenschrift 70: 2646-2652.

Snacken, Sonja (2015) Human Dignity and/or Human Rights for prisoners? Neue Kriminalpolitik 27: 181-189.

Statistisches Bundesamt (Destatis) (2018) Rechtspflege, Strafgerichte. Fachserie 10, Reihe 2.3. Wiesbaden.

Statistisches Bundesamt (2019) Strafverfolgungsstatistik - Sonderauswertung zur Strafverfolgungsstatistik, Personen mit Untersuchungshaft, nach Staatsangehörigkeit Deutsch / Nicht Deutsch nach Ländern, für die Berichtszeiträume 2014 und 2017. Unveröffentlichtes Papier auf Anfrage des NDR, einsehbar bei Verf.

Roxin, Claus \& Schünemann, Bernd (2017) Strafverfahrensrecht. 29. Aufl., München: C. H. Beck. Simon, Jonathan (2007): Governing through crime. Oxford/New York: Oxford University Press. Tyler, Tom (2006) Why people obey the law. With a new afterword. Princeton: Princeton University Press. (Original 1990)

Wacquant, Loic. (2009) Punishing the Poor: The Neoliberal Government of Social Insecurity. Durham / London: Duke University Press.

Weigend, Thomas (2001) Unverzichtbares im Strafverfahrensrecht, Zeitschrift für die gesamte Strafrechtswissenschaft 113: 271-305

Wittig, Petra (2019) Ökonomisierung des Rechts, S. 275-290 in C. Boulanger, J. Rosenstock \& T. Singelnstein (Hrsg.), Interdisziplinäre Rechtsforschung. Wiesbaden: Springer.

Wohlers, Wolfgang (2010) Das Strafverfahren in den Zeiten der „Eilkrankheit“. Neue Juristische Wochenschrift 63: 2470-2475. 